\title{
Psychotherapie der Schlafstörungen
}

Klaus Junghanns

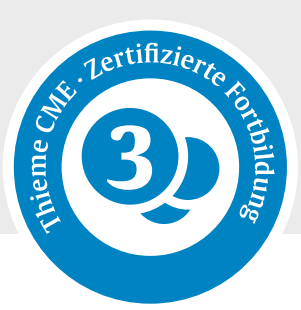

\begin{abstract}
Schlafstörungen treten im Verlauf des Alterns vermehrt auf und neigen zur Chronifizierung. Mithilfe der Kognitiven Verhaltenstherapie der Insomnie (CBT-I) lassen sich nichtorganische Schlafstörungen in ihrer Entstehung und Aufrechterhaltung besser verstehen und mit gezielten Interventionen mildern oder beseitigen. Es ist oft die bessere Alternative zur langzeitigen Verschreibung von Schlafmitteln.
\end{abstract}

\section{Einführung}

Schlaf gehört zum Leben wie Tag und Nacht. Und wie Tag und Nacht ist auch der Schlaf der Mit- oder Gegenspieler des Tageserlebens und der Tagesaktivität. So zeigen sich bei Patienten mit einer Posttraumatischen Belastungsstörung (PTBS), die unter Flashbacks leiden, sehr oft auch Alpträume als Äquivalente einer unzureichenden Traumabearbeitung [1]. Schlafphysiologische Untersuchungen haben gezeigt, dass die Gedächtniskonsolidierung erheblich von komplexen Schlafmechanismen in der Nacht profitiert und dass auch unser Immunsystem und unser metabolisches System einen gesunden Schlaf benötigen, um optimal funktionieren zu können [2, 3]. Insomnie kann diese Funktionen negativ beeinflussen [4]. In den verschiedensten zirkadianen Rhythmen, die unsere Gesundheit wesentlich begleiten, sind ausgeprägte Veränderungen über die Schlafzeit im Vergleich zum Tag auszumachen [5]. Und auch auf der Erlebnisebene kann wohl jeder Mensch bestätigen, dass ein erholsamer Nachtschlaf ihn sehr viel zuversichtlicher und tatkräftiger in den Tag starten lässt. Wenn auch noch nicht geklärt ist, warum wir denn den Schlaf so dringend brauchen, ist es doch auf der Erlebnisebene kein Zweifel, dass er erheblich zu unserem Wohlbefinden und unseren Leiden beitragen kann.

Merke

Guter Schlaf befördert unter anderem das Gedächtnis, die Stimmung, unsere Immunabwehr und unsere emotionale Verarbeitung. Schlechter Schlaf macht das Gegenteil.

Gesunder Schlaf weist eine ultradiane Rhythmik auf. Wir unterscheiden mittels schlafpolysomnografischer Messungen heutzutage 4 Schlafstadien, die wir im Verlauf der Nacht durchwandern [6]: Ein Stadium sehr leichten Schlafes, aus dem wir rasch wieder erwachen können und oft nicht merken, dass wir uns in ihm befinden (N1). In diesem kommt es zu einer assoziativen Lockerung unserer Gedankengänge. Im EEG sieht man eine Rhythmusverlangsamung und ein vermehrtes Auftreten von langsameren
Wellen, den Theta-Wellen. Ihm folgt üblicherweise rasch ein tieferes Schlafstadium (N2), in welchem wir durch Geräusche deutlich weniger leicht erweckbar sind. In diesem Schlafstadium treten Schlafspindeln und K-Komplexe auf, die eine reflektorische Reaktion des Cerebrums auf Geräusche darstellen. Das 3. Schlafstadium (N3) ist gekennzeichnet durch das zunehmende Vorherrschen von Delta-Wellen, die mit 1-2 Hz pro Sekunde auftreten und hochamplitudig im Elektroenzephalogramm (EEG) imponieren. Das 4. Schlafstadium ist gekennzeichnet durch ein ähnliches Hirnstrommuster wie im Schlafstadium N1. Aber im Gegensatz zu diesem treten hier nicht langsame gleitende („schwimmende“) Augenbewegungen auf, sondern schnelle Augenbewegungen (Rapid Eye Movements, REM). Außerdem kommt es in diesem Schlafstadium üblicherweise zu einer zentralen Lähmung der Willkürmotorik, was sich an einem sehr niedrigen Muskeltonus ablesen lässt. In diesem sogenannten REM-Schlaf finden die meisten unserer lebhaften Träume statt. Über die Nacht durchlaufen gesunde Schläfer 4- bis 6-mal einen sogenannten NonREM-REM-Zyklus, d. h. der Schlaf wechselt von N1 über N2 in N3 und dann in eine REM-Schlafphase. Ein einzelner solcher Zyklus dauert meist ca. 60-90 Min, bevor dann ein nächster Zyklus anschließt. Dabei nimmt die Zeit, die man in N3 verbringt, im Verlauf ab, während die Zeit, die man in REM verbringt, zunimmt und typischerweise gegen Morgen am längsten ist ( $\triangleright$ Abb. 1).

\section{Merke \\ In einer Nacht durchwandert ein gesunder Schläfer 4-6 NonREM-REM-Zyklen.}

Parallel zur Schlafrhythmik haben auch eine ganze Reihe an zirkadianen Faktoren einen Einfluss auf den Schlaf. So steigt die Sekretion von Wachstumshormon in den ersten Stunden der Nacht stark an [7]. Es wurde in Zusammenhang mit erholsamem Schlaf, aber auch mit einer effektiven Gedächtniskonsolidierung gebracht. Aber auch die Melatoninsekretion steigt in 


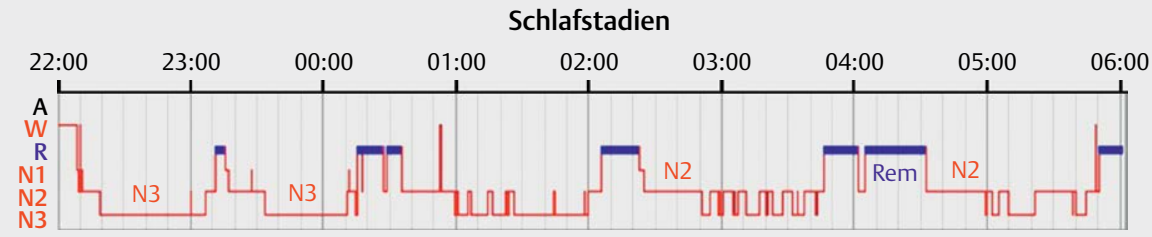

Abb. 1 Schlafverlauf eines gesunden 29-jährigen Studenten. Man erkennt 5 NonREM-REM-Zyklen mit einer Zunahme der REM-Schlafes von der 1. zur 2. Nachthälfte, während in der 1. Nachthälfte der Tiefschlaf (N3) dominanter ist als in der 2. Nachthälfte.

dieser Zeit natürlicherweise an und gilt als wichtiger Zeitgeber auch für die Schlafinitiierung. Die Cortisolsekretion fällt vom Aufstehen über den Tag bis zum Abend allmählich ab und erreicht meist kurz nach Mitternacht ihr Tagesminimum [8]. Es gibt Studien, die eine aktive Inhibierung in dieser Phase fanden [9].Zum Morgen dagegen steigt die Cortisolsekretion stark an und erreicht physiologischerweise um die Zeit des Aufstehens herum ihr Tagesmaximum [10]. Gleiches gilt für die physiologische Körperkerntemperatur, deren Werte zum Abend hin absinken und mit dem Zubettgehen weiter abfallen.

Mit einem niedrigen Cortisol, einem hohen Wachstumshormon- und Melatonin-Ausstoß in der Zeit vor Mitternacht könnte man überlegen, ob die Zeit vor Mitternacht nicht der physiologisch erholsamste Teil der Nacht sein könnte. Diese Meinung ist allerdings bislang nicht wissenschaftlich untermauert. Es ist vielmehr wahrscheinlich, dass Menschen, die gern früh zu Bett gehen und solche, die es vorziehen, später zu Bett zu gehen, versetzte zirkadiane Rhythmen haben. Ein frühes Löschen des Lichtes und Schlafen lässt sich in jedem Fall nicht erzwingen, sodass der physiologische Rhythmus bei den Überlegungen zum Schlaf mit berücksichtigt werden muss. Allerdings lassen sich Schlafgewohnheiten und schlafphysiologische Rhythmen oft nicht sicher trennen. Echte „Lerchen“ oder „Eulen“, die ihren Rhythmus nicht an die sozialen Vorgaben anpassen können, weil ihre zirkadiane Rhythmik dem vollkommen entgegensteht, dürften selten sein.

\footnotetext{
Merke

Die mittlere Schlafdauer gesunder Schläfer liegt bei 7-8 Stunden pro Nacht, aber natürlich gibt es auch viele Menschen, die länger oder kürzer schlafen.
}

Menschen, die sich mit weniger als 6 Stunden Schlaf ausgeruht und erholt erleben, sind eher selten. Man vermutet eine Prävalenz von ca. 1-2\%. Die Zahl derer, die aus beruflichen oder anderen Gründen unter der Woche deutlich weniger als 7 Stunden schlafen, dafür aber am Wochenende länger, ist dagegen deutlich größer.

Patienten beklagen oft eine Unzufriedenheit mit ihrem Schlaf, ohne dies näher zu erläutern. Als erstes wäre hier zu klären, ob die Beschwerden das Ein- oder Durchschlafen betreffen oder ob es sich um unerwünschte und unpassende
Verhaltensauffälligkeiten handelt, die überwiegend aus dem Schlaf heraus auftreten. Bei Ersteren handelt es sich um Dyssomnien, bei Letzteren um Parasomnien. Zu diesen Letzteren gehören u. a. das Schlafwandeln (Somnambulismus), der Pavor nocturnus und die REM-Schlaf-Verhaltensstörung. Zu den Dyssomnien gehört u. a. die Narkolepsie, die Schlafapnoe-Syndrome (obstruktiv und zentral) und das Restless-Legs-Syndrom. In > Tab. 1 sind wesentliche Differenzialdiagnosen bei Schlafstörungen zusammengefasst.

Wichtige differenzialdiagnostische Überlegungen betreffen Schlafstörungen aufgrund anderer Erkrankungen oder Medikationen. So können Schmerzen, Herz-, Lungen-, Leberoder Nierenerkrankungen den Schlaf erheblich stören. Auch eine Reihe von Medikamenten, die im Rahmen dieser Erkrankungen zum Einsatz kommen, kann den Schlaf erheblich stören, wie z. B. Cortison-Präparate oder Antiasthmatika. Und natürlich können auch Drogen wie Alkohol, Amphetamine und Kokain den Schlaf chronisch stören.

Auch Antidepressiva können den Schlaf negativ beeinflussen. So können selektive Serotonin-WiederaufnahmeHemmer (SSRIs) und insbesondere Mirtazapin ein RestlessLegs-Syndrom erheblich verschlechtern oder sogar erst auslösen und so zu erheblichen Einschlafstörungen führen. Aktivierende Antidepressiva wie Bupropion, Duloxetin und Venlafaxin können besonders in hohen Dosen Insomnien befördern, auch wenn sie am Morgen verabreicht werden. Fast alle Antidepressiva wirken REM-suppressiv. Sie können im Verlauf aber lebhaftes Träumen oder Alpträume am Morgen befördern.

Einen großen Teil der Dyssomnien stellen allerdings die Ein- oder Durchschlafstörungen (Insomnien) ohne eine offensichtliche organische Störungsursache dar, die nichtorganischen Insomnien nach ICD-10 (F51.0). Unterschied man früher noch zwischen primären und den sekundären Insomnien, die im Rahmen anderer Störungen, z. B. Rahmen einer Depression, auftreten und nach Abklingen der Stimmungsbeeinträchtigung persistieren, so werden heute alle Insomnien in den neuesten Klassifikationssystemen des DSM-5, der ICSD-3 und der ICD-11 in einer Gruppe zusammengefasst. 
- Tab. 1 Auswahl häufiger Schlafstörungen.

\begin{tabular}{|c|c|}
\hline Diagnose & Symptome (in Stichworten) \\
\hline Restless-Legs-Syndrom (RLS) & $\begin{array}{l}\text { Einschlafstörung, Bewegungsunruhe anfangs in den Beinen, zum Abend verstärkt auftretend. Oft kombiniert mit } \\
\text { periodischen Beinbewegungen im Schlaf, die selbst zu Erwachen führen können. }\end{array}$ \\
\hline $\begin{array}{l}\text { Obstruktives Schlafapnoe- } \\
\text { Syndrom }\end{array}$ & $\begin{array}{l}\text { Eine hohe Zahl an Atemaussetzern im Schlaf aufgrund einer Verlegung der oberen Atemwege, besonders in Rückenla- } \\
\text { ge, oft unerholsamer Schlaf. }\end{array}$ \\
\hline Narkolepsie & $\begin{array}{l}\text { Einschlafattacken, vor oder nach dem Schlaf auftretende (hypnagoge oder hypnopompe) Halluzinationen, Schlafläh- } \\
\text { mungen, Kataplexien (d. h. ein emotionsbedingter Tonusverlust in einzelnen Muskelgruppen), automatisches } \\
\text { Verhalten, unerholsamer Schlaf. }\end{array}$ \\
\hline Alpträume & Aufwachen mit Erinnerung an das Geträumte, oft mit vegetativen Symptomen. \\
\hline Schlafwandeln & $\begin{array}{l}\text { In der ersten Nachthälfte komplexe Bewegungsabläufe im Schlaf, auch mit Reden, ohne Erinnerung am Morgen. } \\
\text { Beginn im Kindesalter typisch. }\end{array}$ \\
\hline Pavor nocturnus & $\begin{array}{l}\text { Ähnlich Schlafwandeln, aber ausgeprägter emotionaler Ausdruck, ebenfalls Amnesie am Morgen, ebenfalls mit } \\
\text { Beginn im Kindesalter. }\end{array}$ \\
\hline $\begin{array}{l}\text { REM-Schlaf-Verhaltens- } \\
\text { störung }\end{array}$ & $\begin{array}{l}\text { Motorisches Ausleben von Aktionen im Traum mit Erinnerung an das Geträumte; typischerweise in der zweiten } \\
\text { Nachthälfte. Ausdruck einer hirnorganischen Störung, deshalb überwiegend nach dem 50. Lebensjahr auftretend. }\end{array}$ \\
\hline
\end{tabular}

\section{Diagnostik}

Bevor wir von einer Insomnie sprechen können, muss neben einer Ein- oder Durchschlafstörung auch eine negative Konsequenz für den Tag bestehen - ein nicht erholsamer Schlaf mit Tagesmüdigkeit und erhöhter Erschöpfbarkeit als Folge der Schlafstörung. Außerdem wird für eine solche Diagnose gefordert, dass die Störung mindestens 3-mal pro Woche auftreten und über einen Zeitraum von 3 Monaten (in der ICD-10 reicht schon ein Monat) bestehen muss. Und die Störung darf nicht durch eine spezielle Ursache wie obstruktive Schlafapnoe, Narkolepsie oder ein RLS erklärbar sein. Die diagnostischen Kriterien für eine Insomnische Störung gemäß des DSM- 5 sind in der nachfolgenden Tabelle zusammengestellt. Diese Kriterien sind präziser als in ICD-10 formuliert.

\section{HINTERGRUNDWISSEN}

\section{Insomnische Störung}

Diagnostische Kriterien einer Insomnischen Störung nach DSM 5 ([11], hier leicht modifiziert)

A. Eine vorherrschende Unzufriedenheit mit der Schlafmenge oder Schlafqualität, die mit mindestens einem der folgenden Symptome assoziiert ist:

- Einschlafschwierigkeiten,

- Durchschlafschwierigkeiten

- Zu frühes Erwachen und Unfähigkeit, wieder einzuschlafen

B. Die Schlafstörung führt zu klinisch relevantem Distress oder Funktionsbeeinträchtigung im Bereich des sozialen, beruflichen, schulischen, akademischen Lebens, im Verhalten oder in anderen wichtigen Funktionsbereichen

C. Auftreten wenigstens 3-mal pro Woche
D. Bestehen über mindestens 3 Monate

E. Die Schlafschwierigkeiten bestehen trotz ausreichender Möglichkeit für Schlaf

F. Die Schlafstörung ist nicht besser durch andere Schlaf-Wach-Störungen wie Narkolepsie, obstruktive Schlafapnoe, zirkadiane Störungen des SchlafWach-Rhythmus oder Parasomnien bedingt und treten nicht ausschließlich unter diesen auf.

G. Die Schlafstörung ist nicht die physiologische Folge einer Substanz, wie z.B. Alkohol, Drogen oder einer Medikation.

H. Komorbide psychische Störungen und medizinische Bedingungen können die vorherrschende Klage über Schlafstörungen nicht angemessen erklären.

Merke

Eine Schlafstörung liegt nur vor, wenn der Schlaf als nicht erholsam erlebt wird und Leidensdruck macht. Die Schlafdauer ist per se ist kein Kriterium für das Vorliegen einer Schlafstörung.

Die diagnostischen Kriterien verlangen lediglich das Erfülltsein subjektiver Kriterien. Eine objektivierende schlafpolysomnografische Untersuchung ist also zur Diagnosestellung nicht erforderlich. Eine solche Untersuchung erlaubt aber den Ausschluss einzelner, nicht anamnestisch zu erfassender Schlafstörungen, wie z. B. Periodische Beinbewegungen im Schlaf, zentrale Schlafapnoen oder Herzrhythmusstörungen, die zu Arousal und darüber zu längeren Wachzeiten führen können. Außerdem hat diese Untersuchung oft eine beruhigende und kognitiv korrigierende Wirkung für die Patienten, da sich üblicherweise zeigt, dass die Betroffenen nach objektiven Kriterien Schlaf haben und oft - verglichen 
- Tab. 2 Abendprotokoll, ein Beispiel.

\section{Abendprotokoll}

\section{Bitte benoten Sie}

Müdigkeit über Tag: 1 = keine Tagesmüdigkeit, 6 = starke Tagesmüdigkeit

Konzentration über Tag: 1 = sehr konzentriert, $6=$ sehr unkonzentriert

Stimmung über Tag insgesamt: 1 = sehr gut, 6 = sehr schlecht

Was glauben Sie, wie Ihr Schlaf in der kommenden Nacht werden wird? 1 = sehr gut, $6=$ sehr schlecht

Tragen Sie ein:

Schlaf am Tag (Nickerchen vorm Fernseher, Mittagsschlaf...) Dauer insgesamt in Min. (geschätzt)

Koffeinhaltige Getränke (Kaffee, Cola, Red Bull) Was, Uhrzeit?

Besonderes am Tage? (bitte angeben)

- Tab. 3 Morgenprotokoll, ein Beispiel.

\begin{tabular}{|l}
\hline Morgenprotokoll \\
\hline Bitte benoten Sie \\
\hline Schlafqualität? 1 = sehr gut, 6 = sehr schlecht \\
\hline Wie erholt fühlen Sie sich jetzt: 1 = sehr gut erholt, 6 = gar nicht \\
erholt \\
\hline Stimmung über Tag insgesamt: 1 = sehr gut, 6 = sehr schlecht \\
\hline Tragen Sie ein: \\
\hline Licht aus (Uhrzeit) \\
\hline Einschlafdauer (geschätzt, in Min.) \\
\hline Wie oft wieder aufgewacht in der Nacht? \\
\hline Dauer des Wachliegezeit insgesamt (grob geschätzt, in Min.) \\
\hline Wann sind Sie morgens aufgestanden (Uhrzeit) \\
\hline Schlafdauer insgesamt (grobe Schätzung) in Stunden und Min. \\
\hline
\end{tabular}

mit den parallel auszufüllenden Schlafprotokollen - mehr als sie erwartet oder eingeschätzt hatten.

\section{Merke}

Schlafstörungen sind selten jede Nacht in gleichem Maße vorhanden und auch die Konsequenzen für den Tag sind entsprechend nicht konstant.

Um eine Insomnie genauer zu analysieren, werden üblicherweise über ein bis mehrere Wochen Schlafprotokolle geführt, in denen in einem Abendprotokoll Aspekte der Tagesaktivität und in einem Morgenprotokoll Aspekte des Schlafes erfasst werden. Um von einer relevanten Schlafstörung sprechen zu können, wird gefordert, dass der Schlaf an zumindest 3 Nächten einer Woche beeinträchtigt ist (siehe Diagnostische Kriterien). Nachfolgend sind Beispiele eines solchen Abend- und Morgenprotokolls aufgeführt ( $\$$ Tab. 2 und $\mathbf{3}$ ).

\section{Störungsmodell der Psychotherapie}

Die etablierteste Form der Psychotherapie der Insomnien erfolgt auf der Grundlage einer kognitiven Verhaltenstherapie der Insomnie (CBT-I, u. a. [12-16]). Sie hat sich als erfolgreich gegenüber einer Wartelisten-Bedingung erwiesen (u. a. [17]) und ist Bestandteil der S3-Leitlinien zu nicht erholsamen Schlaf [18]. Es erfolgten jedoch bislang keine randomisierten kontrollierten Studien hierzu.

Die Psychotherapie der Insomnien geht zum einen von einem Teufelskreis der chronifizierten Schlafstörungen aus: Aversive Emotionen wie Ärger, Angst und Traurigkeit wirken sich negativ auf das Ein- und Durchschlafen aus und stehen im Zusammenhang mit dysfunktionalen Kognitionen zu den Schlafproblemen und ihren Folgen und zu einem psychophysiologischen Arousal. Diese Aspekte zusammen unterhalten die Schlafstörung und führen oft zu einem sozialen Rückzugsverhalten der Betroffenen, welches wiederum die Schlafstörung verschlimmert und die Fokussierung auf den Schlaf erhöht. Dieser Teufelskreis ist nachfolgend dargestellt ( $\mathbf{A b b}$. 2).

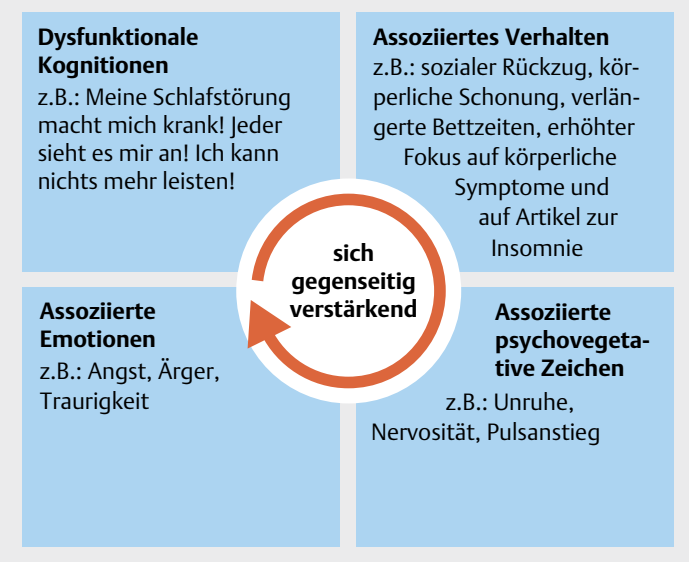

- Abb. 2 Teufelskreis der Schlafstörungen: Gedanken, Emotionen, psychovegetative Aspekte und das Verhalten. 
Eine biografisch hergeleitete Erklärung der Schlafstörung geht vom sogenannten 3P-Modell von Spielman aus [19]. Hier werden die Entstehungsbedingungen der Störung (Predisposing factors), die Erstauftrittsbedingungen (Precipitating factors) und die aufrechterhaltenden Bedingungen (Perpetuating factors) beschrieben. Zu den Entstehungsbedingungen kann eine positive Familienanamnese für Schlafstörungen gehören, aber auch genetische Prädispositionen oder Modelllernen. Auch der Umstand, dass guter Schlaf in der Familie als besonders zentral erachtet wurde, kann den Fokus auf mögliche Schlafprobleme verstärkt haben. Ein unregelmäßiges Schlafverhalten oder schlafhygienisch problematische Verhältnisse, wie Lärm oder lange Fernsehabende, können beitragen. Die Erstauftrittsbedingungen beschreiben die Faktoren, die zum Auftreten der Schlafproblematik beigetragen haben. So beklagen viele Frauen erste Schlafprobleme in der Schwangerschaft oder dem Klimakterium. Auch berufliche oder soziale Veränderungen wie Schichtdienste, Scheidung oder Sterbeprozesse können zur Auslösung der Schlafprobleme beitragen.

\section{Merke}

Viele Menschen haben im Verlauf ihres Lebens gelegentlich Schlafprobleme. Diese sind meist zeitlich begrenzt und lösen sich schließlich wieder auf.

Wenn Schlafprobleme chronifizieren, findet man oft Verhaltensweisen, die die Schlafstörung ungewollt stabilisieren. Es kommt also zu Verhaltensweisen, die Folge der Schlafstörung sind und scheinbar der Anpassung an das Problem dienen, aber die dennoch die Störung letztlich verstärken bzw. stabilisieren. So kann sozialer Rückzug erfolgen, weil man meint, sich schonen zu müssen oder jederzeit in der Lage sein zu können, ins Bett zu gehen. Dieses Schonverhalten sorgt aber für einen Verlust von angenehmen Aktivitäten und dadurch zu einem Verlust positiver Verstärker. Alkohol kann als Schlafmittel eingesetzt werden, obwohl es zwar das Einschlafen fördert, aber das Durchschlafen und das Erholtsein am Morgen negativ beeinflusst. Auch Schlafmittel können nach anfänglicher positiver Wirkung zu einer Toleranzentwicklung mit Dosissteigerung und schließlich Abhängigkeit führen. Dies trifft besonders auf die Benzodiazepine und die sogenannten Z-Präparate zu (Zolpidem, Zopiclon und Zaleplon). Aber auch verlängerte Bettzeiten, Grübeln um die Folgen des verminderten Schlafes u.v.m. können letztlich zur Aufrechterhaltung des Schlafproblems beitragen. Insbesondere die individuelle Analyse dieser aufrechterhaltenden Bedingungen kann sehr hilfreich sein, um die psychotherapeutische Behandlung an die Bedürfnisse des Betroffenen anzupassen.

\section{Merke}

Die kognitive Verhaltenstherapie der Insomnie setzt vorwiegend an den Mechanismen an, die zu einer Chronifizierung der Schlafprobleme führten, den aufrechterhaltenden Bedingungen.

\section{FALLBEISPIEL}

Die 66-jährige verwitwete Frau B. berichtet, dass sie schon seit 2 Jahren vermehrt Ein- und Durchschlafstörungen habe. Erstmals seien die Schlafprobleme aufgetreten, als ihr Mann vor 15 Jahren unerwartet verstorben sei. Danach habe sie „ein Jahr gar nicht geschlafen“. Schließlich habe sie von einem Psychiater Trimipramin verschrieben bekommen, welches sie bis vor 2 Jahren eingenommen habe, ohne dass sie eine ausgeprägte Verbesserung gehabt habe. Auch Melatonin und Baldrian habe sie danach ausprobiert, ohne dass der Schlaf sich wesentlich verbessert habe. Sie sei schon froh gewesen, wenn sie auf 5 Stunden Schlaf gekommen sei. An 6 von 7 Tagen sei der Schlaf jedoch unter 5 Stunden gewesen. Sie benötige regelhaft 1-2 Stunden, um einzuschlafen; 2-3 Stunden später sei sie dann das erste Mal wieder wach und schlafe dann bis in den Morgen noch etwa 2 Stunden in kurzen Schlaf- bei langen Wachintervallen. Sie gehe gegen 22.00 Uhr zu Bett und stehe gegen 7.30 Uhr wieder auf, fühle sich stets unerholt und sei den Tag über beständig müde, was zum Abend hin mehr wird. Trotzdem sei sie leistungsfähig, es koste sie aber viel Anstrengung. Sie habe deshalb ihre sozialen Kontakte, insbesondere zum Abend hin reduziert und gehe bspw. nicht mehr ins Theater, um möglichst keinen Schlaf zu verpassen. Abends sei sie todmüde und fühle sich doch zugleich auch ein wenig unruhig und überdreht. Wenn sie dann am Fernseher einnicke, stehe sie auf und gehe zu Bett. Wenn sie sich aber fürs Schlafen fertig mache, werde sie wieder stärker unruhig und fühle sich wach, wenn sie ins Bett steige. Hinweise auf ein Restless-Legs-Syndrom oder eine Narkolepsie ergeben sich nicht. Eine ambulante Schlafuntersuchung hatte keinen Hinweis auf eine Schlafapnoe erbracht. Sie rauche wenig, trinke selten Alkohol, nehme keine Drogen ein, trinke morgens 2 Becher Kaffee, am Mittag einen Espresso. In den letzten beiden Jahren kümmere sie sich vermehrt um ihre dementen Eltern und um ihren Bruder, der ausgeprägte Eheprobleme habe. Sportlich sei sie sehr aktiv, mache aber zum Abend hin keinen Sport. Wenn sie etwas psychisch belaste, fühle sie sich allerdings sehr aufgedreht. Körperlich sei sie bis auf eine Arthrose in der linken Schulter gesund. Schulterschmerzen würden sie nicht beeinträchtigen. Aktuell nehme sie keinerlei Medikation ein. Aus der Familienanamnese ist bekannt, dass auch die Mutter eine schlechte Schläferin gewesen ist. Aus dieser Vignette wird deutlich, dass Frau B. von den Entstehungsbedingungen her familiär vorbelastet ist und dass die Schlafstörung chronisch 
geworden ist. Während sie 9,5 Stunden im Bett verbringt, kommt sie in dieser Zeit subjektiv nur auf 5 Stunden Schlaf. Sie hat regelhaft eine lange Einschlaflatenz, aber auch das Durchschlafen ist erheblich beeinträchtigt. Bei ausgeprägter Tagesmüdigkeit kommt sie vor dem Zubettgehen in eine psychovegetative Erregungssituation, die an eine konditionierte Reaktion in Bezug auf das Bett und die damit verbundene Schlafstörung schließen lässt. Wie die Durchschlafstörung aufrechterhalten wird, ist unklar. Eine zu lange Bettzeit könnte hierfür mit verantwortlich sein. Ihr sozialer Rückzug ist Ausdruck eines Schonverhaltens und führt zu einem Verstärkerverlust.

\section{Therapie mittels CBT-I}

\section{Behandlungselemente}

Die psychotherapeutische Behandlung einer chronischen Schlafstörung lässt sich in 8 Behandlungselemente untergliedern:

1. Dokumentation der Therapie, des Schlafes und seiner Veränderungen

2. Psychoedukation zum Schlaf und Überblick über die Therapie

3. Schlafhygiene

4. Erlernen und Umsetzung eines Entspannungsverfahrens

5. Stimuluskontrolle

6. Schlafrestriktion

7. Bearbeitung dysfunktionaler Gedanken

8. Ergänzende Maßnahmen

Die Behandlungserfolge der CBT-I beziehen sich überwiegend auf nichtorganische Insomnien, deren Ursache nicht in einer psychischen Störung wie etwa einer Depression liegt. Typische Therapielängen liegen bei einer Sitzung pro Woche und 5-12 Sitzungen. Das Setting ist einzel- oder gruppentherapeutisch, wobei sich ein einzeltherapeutisches Setting wegen der Möglichkeit des persönlicheren Zuschnittes der Therapie als besser erwiesen hat. Schließt man auch sekundäre Insomnien ein, wird es sinnvoll sein, auch längere Therapielängen ins Auge zu fassen. Insbesondere für die Bearbeitung dysfunktionaler Gedanken ist erfahrungsgemäß eine mindestens 4-wöchige Bearbeitung sinnvoll, um andauernde Veränderungen in den Kognitionen zu erreichen.

\section{PRAXISTIPP}

Eine ausschließlich auf die Insomnie ausgerichtete Therapie kann binnen 5-12 Sitzungen eine deutliche Verbesserung des Schlafes erreichen. Bei komorbiden Störungen kann ein zeitweiliger Fokus auf den Schlaf sinnvoll sein, um die Gesamtproblematik positiv zu beeinflussen.
Die 8 benannten Elemente sollen nun im Einzelnen dargestellt werden.

\section{Dokumentation der Therapie, des Schlafes und seiner Veränderungen}

Die Schlafprotokolle sind ein fester Bestandteil der Therapie. Sie erlauben es, die Einhaltung der abgesprochenen Verhaltensmaßnahmen und die Fortschritte im Verlauf zu überprüfen. Sie haben auch eine wesentliche Bedeutung in der Bearbeitung dysfunktionaler Gedanken. Dabei ist es hilfreich, die extratherapeutischen Veränderungen zusätzlich abzufragen, da diese einen erheblichen Einfluss auf die Therapie haben können. So können bspw. Schmerzen im Rahmen einer körperlichen Erkrankung oder Medikamentenumstellungen oder auch Veränderungen im sozialen Kontext wie Scheidung, Erziehungsprobleme, Umzug, neue Partnerschaft, Berufswechsel oder Probleme im Beruf sehr relevante Faktoren sein, die erhebliche Auswirkungen auf den Schlaf mit sich bringen, im Guten wie im Schlechten. Die schnelle Erfassung der extratherapeutischen Bedingungen ist in dem nachfolgenden Kurzfragbogen beispielhaft wiedergegeben, der von den Patienten unmittelbar vor jeder Sitzung ausgefüllt werden kann.

\section{FRAGEBOGEN}

\section{Extratherapeutische Veränderungen}

Bitte kreuzen Sie an, ob sich in folgenden Bereichen seit der letzten Sitzung wesentliche Veränderungen ergeben haben:

- Medikamenten-Umstellungen

- Veränderungen im Berufsleben (Arbeitsunfähigkeit, Berentung, Berufswechsel, Veränderungen der beruflichen Atmosphäre...)

- Veränderungen im Privatleben (Partnerschaft, Schlafsituation, Erziehungsprobleme, Freundeskreis, Krankheiten...)

- Sonstiges, nämlich:

- Keine Veränderungen seit der letzten Sitzung

Merke

Auch in der Bearbeitung von Schlafproblemen sollte man extratherapeutische Einflüsse mit im Blick haben.

Ein ebenfalls unspezifischer, aber sehr relevanter Wirkfaktor in der Therapie ist das Feedback des Patienten. Dieses sollte wiederholt eingeholt werden, um zu schauen, inwieweit der Patient sich verstanden gefühlt hat und von der jeweiligen Sitzung profitierte und ggf. warum nicht.

Hierzu sollte am Ende jeder Sitzung vom Patienten eine kurze Zusammenfassung des Besprochenen erbeten werden im Sinne eines Teach-back und der Patient sollte die Relevanz des Besprochenen für seine Schlafproblematik bewerten. Auch der Inhalt und der Sinn der Hausaufgaben 
sollten kurz diskutiert werden. Abschließend sollte man sich nicht scheuen, aktiv zu erfragen, ob der Patient sich in der abgelaufenen Sitzung angemessen in seiner Problematik gesehen (validiert) und verstanden fühlte. Die therapeutische Allianz in Bezug auf das therapeutische Vorgehen kann hierdurch besser überprüft und ggf. nachgebessert werden. Sie ist ein wesentlicher Wirkfaktor in der Psychotherapie [20].

\section{Psychoedukation zum Schlaf}

Es ist sehr hilfreich für die Betroffenen, Informationen über den Schlaf zu bekommen, da viele Betroffene verzerrte Vorstellungen davon haben, was guter Schlaf bedeutet. So ist es wichtig deutlich zu machen, dass der Schlaf sich über die Lebensspanne verändert. Es ist nicht ungewöhnlich, wenn man mit zunehmendem Alter wiederholt kurz wach wird oder wasserlassen muss. Auch wird der Schlaf eher oberflächlicher im Verlauf. Der Tiefschlaf nimmt mit dem Alter ab, insbesondere bei Männern. Es ist wichtig zu wissen, dass Gedanken kein Beleg sind dafür, dass man wach ist. Im Gegenteil ist es möglich, darüber nachzudenken, wie der nächste Tag wird, wenn man jetzt nicht schläft, und tatsächlich schläft man! Gerade im Schlafstadium N2 können solche kognitiven Prozesse stattfinden. Dies ist einer der Gründe dafür, dass viele Menschen ihre tatsächliche Schlafdauer unterschätzen.

\section{PRAXISTIPP}

Das Wissen darum, dass der Schlaf länger ist, als man dachte, kann für einen Menschen mit Insomnie sehr beruhigend sein, weshalb eine schlafpolysomnografische Untersuchung in einem Schlaflabor sehr hilfreich sein kann.

Wichtig ist auch, darauf hinzuweisen, dass ein Mittagsschlaf in die Berechnung der Schlafdauer mit eingehen muss. Wer eine Stunde am Mittag schläft, sollte sich nicht wundern, dass er in der Nacht eine Stunde weniger Schlaf benötigt. Auch der Umstand, dass Menschen unterschiedlich viel Schlaf benötigen und dass es nicht sinnvoll ist, die Schlafdauer an der des Partners auszurichten, kann hilfreich sein. Menschen mit Schlafproblemen in der Nacht sollten möglichst auf Mittagsschlaf verzichten oder ihn möglichst kurz halten (sog. Powernap, bis eine halbe Stunde). Schon ein Daliegen mit geschlossenen Augen kann zu einem Schlaf führen, der nur nicht als solcher wahrgenommen wird. Die Betroffenen sollten deshalb auch eine solche Form des Ausruhens lassen oder aber am Tage möglichst kurz halten.

Merke

Mittagsschlaf und ein Ausruhen am Tag können Einschlafprobleme befördern und sollten in den Gesamtschlaf mit einberechnet werden.

\section{Schlafhygiene}

Es gibt eine Vielzahl an möglichen Störfaktoren für den Schlaf. Hierzu gehört Straßen- oder Fluglärm, ein zu warmes oder zu kaltes Zimmer, der Konsum von Alkohol oder Drogen und Nikotin, stimulierende Getränke. Natürlich kann ein schnarchender Bettpartner oder Lärm in der Wohnung aufgrund stark abweichender Bettzeiten zwischen den Mitbewohnern ein Problem für den Schlaf sein.

Merke

Regelmäßige Bettzeiten sind eine Grundvoraussetzung für eine positive Veränderung des Schlafes.

Sehr stark wechselnde Bettzeiten können erheblich zur Persistenz der Schlafstörung beitragen. Dies ist auch einer der Gründe, warum durch Schichtdiensttätigkeiten Insomnien entstehen können [21]. Wichtig ist v. a., dass man seine Aufstehzeit recht stabil hält. Man sollte also möglichst nicht die Bettzeit in den Tag hinein ausweiten, um fehlenden Schlaf aus der Nacht wettzumachen. Dies führt eher dazu, dass die Schlafproblematik sich auch in der nächsten Nacht fortsetzt, während die in Kauf genommene Tagesmüdigkeit nach einer schlechten Nacht die Chancen für eine erholsamere nachfolgende Nacht erhöht. Eine Auswahl an schlafhygienischen Regeln ist im Kasten dargestellt. Wir motivieren Patienten immer wieder, Dinge auszuprobieren und kreativ zu sein bei der Suche nach guten Rahmenbedingungen für den Schlaf.

SCHLAFHYGIENISCHE EMPFEHLUNGEN

(AUSWAHL)

- Möglichst ruhiges, dunkles, etwas kühleres Schlafzimmer

- Keine schweren Mahlzeiten am Abend

- Keine größeren Trinkmengen am Abend

- Kein Alkohol, keine Stimulanzien (Tee, Kaffee, koffeinhaltige Softdrinks) nach 16.00 Uhr, möglichst kein Nikotin am Abend, keine Drogen

- Keine aufregenden Tätigkeiten vor dem Zubettgehen, keine Arbeit am PC

- Ausdauersport und Spaziergänge sind gut, sollten nicht zu spät erfolgen

- Entspannung und Herunterfahren vor dem Zubettgehen

- Am Abend keine sozialen Netzwerke mehr nutzen

- Keine Arbeit mit ins Bett nehmen, kein Fernsehen im Bett

- Regelmäßige Bettzeiten

Menschen neigen zu Ritualen. Es gibt Trauerrituale, Aufstehrituale, Feierrituale und viele mehr. In gleicher Weise sollte es auch ein Ritual für Schlafgestörte am Abend geben. Dazu gehört es, vor dem Zubettgehen den Tag 
ruhig ausklingen zu lassen. Da viele Patienten mit Insomnie beklagen, dass sie „nicht abschalten“ können und gedanklich noch mit Sorgen oder Analysen dessen, was am Tag passiert ist oder am nächsten Tag ansteht, beschäftigt sind, ist es hilfreich einen „Sorgenstuhl“ einzuführen. Hier geht es darum, einen Ort außerhalb des Schlafzimmers zu wählen, wo man sich hinsetzen und seine Gedanken niederschreiben kann. An diesem Ort darf man grübeln und analysieren, am besten in einer Zeit etwas vor der Bettzeit. Der Auftrag ist es, diese Sorgen dann in dem Heft oder auf jenem Papier bis zum Ende der Nacht zu belassen. Im Falle von weiteren solcher Gedanken nach dem Zubettgehen soll man aufstehen, um diese Gedanken dort am Sorgenstuhl niederzuschreiben und sie dort zurücklassen, in dem Wissen, dass man sie jederzeit am nächsten Tag wieder aufsuchen kann.

\section{PRAXISTIPP}

Eine Grübel- oder Sorgenstunde einzurichten kann dazu beitragen, Grübeln und Sorgen zu reduzieren und insbesondere aus der Nacht zu nehmen.

Auch das Durchgehen positiver Erlebnisse des Tages kann ein hilfreiches Ritual sein, um besser zur Ruhe zu kommen. In einem angenehmen Buch zu lesen (möglichst außerhalb des Bettes) und sich einen beruhigenden, genussreichen Tee zu machen oder einen kleinen Spaziergang zu machen, können hilfreich sein. Es gibt eine unendliche Zahl an Möglichkeiten, den Tag angenehm zu beenden und so entspannt die Nacht im Bett anzugehen, und jeder Mensch hat ein Gespür dafür, was für ihn besonders hilfreich ist.

\section{Merke \\ Schlafhygienische Empfehlungen sollten nicht als unangenehme Forderung oder Verpflichtung vermittelt werden, sondern als Anregungen, die man ausprobieren sollte}

Überhaupt sollte man den Betroffenen stets Gelegenheit geben, sich selbst einzubringen in die Überlegungen zur Schlafhygiene. Es geht ja darum, dass die Betroffenen das für sie Hilfreichste entwickeln und umsetzen. Wenn jemand bspw. durch Rockmusik besonders gut zur Ruhe kommt und sich dies in den Schlafprotokollen auch als hilfreich abbildet, soll der Betroffene dies nutzen. Er sollte aber auch Abstand davon nehmen können, wenn sich in den Protokollen zeigt, dass dies nicht hilft oder sogar negative Auswirkungen zu haben scheint.

Erlernen und Umsetzen eines

Entspannungsverfahrens

Um die physiologische Erregung herunterzufahren, hat sich bei Schlafstörung insbesondere die Progressive Muskelentspannung (PME) als hilfreich erwiesen. Dies wird zu- nächst außerhalb des Bettes im Sitzen erlernt. Wir bieten üblicherweise eine Sitzung von 20-30 Min. an, da diese von den Patienten in der Regel gut angenommen wird. Längere Sitzungen werden zuhause meist nicht umgesetzt.

\section{Merke \\ Progressive Muskelentspannung kann den Patienten beim Einschlafen helfen.}

Im Rahmen der PME werden einzelne Muskelpartien eine Zeitlang leicht angespannt und anschließend wieder entspannt. Der Betroffene wird angehalten, sich in diese reaktive Entspannung gezielt einzufühlen. Typischerweise wird im Bereich der Hände begonnen und dann der gesamte Köper systematisch durchgegangen. Die Entspannung wird mit einem Ruhebild abgeschlossen, in welchem sich der Patient einen Ort imaginieren soll, der für ihn ein Inbegriff von Entspannung ist. Er soll diesen Ort allein aufsuchen und die Imagination wird durch Ansprechen der verschiedenen Sinneskanäle intensiviert (Was fühlen Sie auf der Haut? Was riechen Sie, was sehen Sie, was hören Sie dort? Vielleicht schmecken sie auch etwas auf der Zunge?).

Die PME wird zunächst im Sitzen angeleitet und soll täglich geübt werden, bis der angestrebte Effekt einer Entspannung zuverlässig erreicht werden kann. Dann soll die PME auch im Bett zur Einschlafförderung eingesetzt werden. Gute Effekte lassen sich auch mit einer Form von Vorsatzbildung erreichen: Der Betroffene soll sich vor dem Zubettgehen z. B. vornehmen und sagen: „Wenn ich in der Nacht merke, dass ich dabei bin, wach zu werden, werde ich meine Hände kurz anspannen, mir das Ruhebild vor Augen führen und dann mit einem tiefen Ausatmen die Hände entspannen und mit dieser Entspannung zurück in den Schlaf gleiten“.

Progressive Muskelentspannung hat sich als lernbar binnen 1-2 Wochen und wirksam erwiesen. Es spricht jedoch nichts dagegen, wenn ein Patient autogenes Training erlernt hat und dieses vorzieht.

Die schlafentspannende Maßnahme sollte in einem Tagesprotokoll dokumentiert werden. Es ist sinnvoll, die Anspannung vor und nach der Übung zu dokumentieren, um die Wahrnehmung für die entspannende Wirkung zu befördern.

\footnotetext{
Merke

Progressive Muskelentspannung zielt auf eine Senkung der Grundanspannung vor der Bettzeit. Kombiniert mit der Vorsatzbildung kann sie das Ein- und Durchschlafen positiv beeinflussen.
} 


\section{Stimuluskontrolle}

Das Bett hat sich für Insomnie-Patienten oft zu einem Kampfplatz entwickelt: Es wurde zum Ort, an dem sie wiederholt den Kampf um den Schlaf verloren haben und an dem Gefühle von Verzweiflung und Wut oft hochkamen. Viele Betroffene können schildern, wie sie sich sehr müde auf die Schlafzeit vorbereiten, um paradoxerweise im Rahmen des Zähneputzens und Gesichtwaschens eine zunehmende, wachmachende Unruhe zu verspüren, die als Anzeichen dafür genommen wird, dass es mit dem Schlaf wohl nicht sogleich klappen wird, was wiederum negative Auswirkungen auf die Einschlafneigung hat. Die hieran beteiligte klassische Konditionierung soll geschwächt werden, indem der Patient angehalten wird, nicht länger als 30 Min. wach im Bett zu liegen. Wenn er nicht einschlafen kann in dieser Zeit, soll er wieder aufstehen und einer einfachen, wenig Konzentration, psychische und körperliche Aktivität erfordernden Aufgabe nachgehen, wie z. B. zu bügeln oder abzuwaschen. Er sollte gedämpftes Licht nutzen. Erst wenn er sich wieder ausreichend müde fühlt, soll er erneut zu Bett gehen. Sollte er wieder nicht einschlafen, steht er wieder auf usw. Dieses Vorgehen kann man auch beim Wiederaufwachen in der Nacht einsetzen, wobei viele Patienten dies als zu mühevoll erleben. Trotzdem soll er am Morgen zu einer festen Zeit aufstehen und auch keinen zusätzlichen Mittagsschlaf einbauen. Natürlich führt eine solche Maßnahme leicht zu einem Schlafdefizit, welches aber über die nächsten Nächte die Einschlaffähigkeit verbessern soll. Es soll also eine zeitlich befristete Intervention sein. Allerdings sollte der Patient sich angewöhnen, mit Müdigkeit zu Bett zu gehen und nicht aufgrund der Uhrzeit.

\section{Merke}

Stimuluskontrolle zielt v. a. auf Einschlafstörungen, kann aber auch bei Durchschlafstörungen zum Einsatz kommen.

\section{Schlafrestriktion}

Die Schlafrestriktion ist eine effektive Maßnahme bei Durchschlafstörungen und Früherwachen. Ziel ist es, den Anteil des Schlafens an der Bettzeit (Schlafeffizienz) in einen normalen Bereich, d. h. über 85 \% zu bekommen.

In seiner klassischen Form wird mit dem Patienten zunächst die mittlere Schlafeffizienz anhand seiner Schlafprotokolle ermittelt. Die Zeit, die der Patient im Mittel pro Nacht geschlafen hat, wird nun festgelegt als die zukünftige Bettzeit. Das Ziel ist es nun, in dieser verkürzten Bettzeit eine Schlafeffizienz von mindestens $85 \%$ an 3 aufeinander folgenden Tagen zu erreichen. Gelingt dies, wird die Bettzeit um 15 Min. ausgeweitet, bis auch hierbei eine Schlafeffizienz von mindestens $85 \%$ erreicht wird und so weiter bis zu einer Bettzeit, wo diese Schlafeffizienz nicht mehr erreicht werden kann. Fünfzehn Minuten unterhalb der letzten Bettzeit liegt dann die künftige einzuhaltende Bettzeit.
Diese Technik zielt darauf, über den anfangs entstehenden Schlafmangel die Müdigkeit soweit zu steigern, dass die Wahrscheinlichkeit eines intensiveren Schlafes schließlich steigt und die Assoziationen von Bett und Wachliegen abnehmen oder sogar gelöscht werden können.

Diese Technik wird von vielen Patienten als zu anstrengend erlebt, um sie in dieser Form umzusetzen. Man muss bei vielen der Patienten auch davon ausgehen, dass sie ihre Schlafzeit eher unterschätzen und so in den Protokollen eher zu niedrige Schlafmengen dokumentieren. Deshalb wird als weniger restriktive Form folgendes Vorgehen gelegentlich eingesetzt: Der Patient wird befragt, welche Schlafzeit ihm ermöglichen würde, trotz Tagesmüdigkeit halbwegs zurechtzukommen. Diese Zeit wird dann als Bettzeit gesetzt. Die meisten Patienten geben hier 5-6 Stunden an, was den Schlafdruck ausreichend erhöhen sollte.

\section{Merke \\ Schlafrestriktion zielt auf eine Verbesserung der Schlafeffizienz und damit v. a. auf Durchschlaf- störungen.}

\section{Bearbeitung dysfunktionaler Gedanken}

Wenn Patienten in der Nacht wachliegen, geben sie häufig an, dass ihre Gedanken um die negativen Konsequenzen des Wachliegens kreisen, insbesondere um die Folgen für den nachfolgenden Tag. Beispiele sind: „Wenn ich jetzt nicht endlich einschlafe, wird der morgige Tag eine Katastrophe!“ oder „Mein Partner schläft so schnell ein, während ich wachliegen muss. Das ist ungerecht!“ oder „Ich hätte es vorhersehen können, dass diese Nacht wieder kein Schlaf kommt. Ich hätte mich anders verhalten müssen! (Keinen Wein trinken, früher ins Bett gehen, Theater oder Feier absagen etc.)“. Dies sind Kognitionen, die Ängste bzw. Ärger schüren und damit auch physiologische Reaktionen stützen, die schlafstörend sind (Depressive Patienten kreisen in ihren Gedanken übrigens meist um andere Inhalte, nämlich die von Aaron T. Beck beschriebene kognitive Trias: Inhalte, die einen niedrigen Selbstwert, eine negative Bewertung der eignen Person durch die Anderen und eine perspektivische Hoffnungslosigkeit zum Ausdruck bringen).

Es sollte nun herausgearbeitet werden, welche Emotionen diese dysfunktionalen Gedanken generieren und dass diese Emotionen den Teufelskreis der Schlafstörung mit unterhalten. Wenn diese Kognitionen also die Schlafstörung eher aufrechterhalten und sie insofern dysfunktional sind, sollten sie durch funktionalere Kognitionen ersetzt werden. Hierzu kann man die allgemeinen Informationen nutzen, die schon zum Schlaf gegeben wurden. Aber auch mithilfe der Schlafprotokolle lässt sich meist gut herausarbeiten, dass die Kognitionen in ihrer Art der Katastrophisierung der Folgen der Insomnie nicht berechtigt oder überzogen sind. Es lässt sich gut aufzeigen, dass nicht jede 
Nacht gleich schlecht geschlafen wurde und dass auf eine schlechte Nacht nicht automatisch ein schlechter Tag folgt. Und auch die Vorstellung, dass es beim Schlafen gerecht zugehen müsse, erweist sich in praxi als unbegründet und schon gar nicht hilfreich für das Ein- oder Durchschlafen. Auch perfektionistische und dichotome Sichtweisen, was gut und richtig ist, um einen guten Schlaf zu bekommen und was allein ein guter Schlaf ist, stehen einer entspannten, akzeptierenden Grundhaltung entgegen und sind dadurch schlafstörend. Die Infragestellung und Modifikation dieser Überzeugungen und Gedanken kann dazu beitragen, die mit ihnen assoziierten physiologischen und emotionalen Reaktionen zumindest zu mildern und dadurch den Schlaf zu erleichtern. Wenn es schlafpolysomnografische Untersuchungen zum Schlaf der Betroffenen gibt, kann dies zusätzlich dazu beitragen, katastrophisierende Gedanken zu relativieren (siehe oben).

\section{Merke \\ Dysfunktionale Gedanken bezüglich der Folgen des Schlafmangels lassen sich oft anhand der Schlafprotokolle überprüfen.}

Diese kognitive Arbeit ist für viele Patienten sehr ungewohnt und bedarf einer guten Einführung. Das Gewahrwerden der oft automatisch kommenden Gedanken und der mit ihnen verknüpften emotionalen und physiologischen Reaktionen sowie die Suche nach alternativen, funktionaleren Gedanken, will geübt sein und kann oft 4 und mehr Sitzungen in Anspruch nehmen. Insbesondere, wenn eine psychische Komorbidität wie eine Depression, eine Angststörung oder eine anankastische Persönlichkeitsstörung besteht, kann sie aber ein besonders wichtiges Element der Verbesserung sowohl des Schlafes, als auch der komorbiden Störung sein.

\section{Ergänzende Maßnahmen}

\section{Achtsamkeit}

Mit der Dritten Welle der Verhaltenstherapie haben Achtsamkeitsmeditationen Eingang in den Mainstream der Psychotherapie gefunden. Über regelmäßige Achtsamkeitsübungen gelingt es oft, Stress zu reduzieren, sich seiner Gedanken bewusster zu werden und Emotionen und Gedanken besser zu entkoppeln. Aus meiner Erfahrung wird diese Technik von den Betroffenen zwar anfangs skeptisch, letztlich dann aber gut angenommen. Ziel der Meditation ist es, in einer Phase der Ruhe und Stille das gedankliche Abschweifen von einem Fokus wie der Atmung möglichst früh zu erkennen und dann auf den Fokus zurück zu kommen. In diesem Sinne geht es also weniger um Entspannung als vielmehr um das Gewinnen einer Gelassenheit gegenüber den intrapsychischen Abläufen. Da diese Gelassenheit dazu beitragen kann, Sorgengedanken bezüglich der Schlafproblematik zurückzufahren, ist ein indirekter Effekt auf den Schlaf zu erwarten. In der klinischen Pra- xis sehe ich dies bestätigt. Es fehlen jedoch bislang Studien hierzu.

\section{Merke \\ Achtsamkeit kann darin unterstützen, automatische Gedanken zu erkennen und Abstand von diesen und den emotionalen Reaktionen zu bekommen.}

\section{Aktivierung von Ressourcen}

Angenehme Aktivitäten, selbstfürsorgliches Verhalten und eine körperliche und soziale Aktivierung, wie z. B. gemeinsame Unternehmungen mit Freunden oder der Familie, sollten ebenfalls im Fokus der Therapie stehen. Sie wirken stimmungsaufhellend, fördern die abendliche Zufriedenheit und eine angenehme Erschöpfung. Sie können helfen, die Sorgen um den Schlaf ein wenig zu relativieren und dadurch den Stress, der mit einer starken Fokussierung auf optimale Schlafbedingungen verbunden sein kann, zu reduzieren. Der Einbau solcher angenehmen Aktivitäten, der auch Ressourcen erweitert oder wieder aufleben lässt, kann unterstützt werden, indem er in die Hausaufgaben integriert und jedes Mal validierend abgefragt und verstärkt wird.

\section{FALLBEISPIEL}

Frau B. stellte unter der erlernten PME fest, dass sie entspannter ins Bett ging. Unter der Technik der Stimuluskontrolle verkürzte sich die Einschlaflatenz auf zuletzt 20-30 Min. Und unter der Schlafrestriktion erreichte sie letztlich bei einer Bettzeit von 7,5 Stunden eine subjektive Schlafdauer von 6,5 Stunden bei einer Verringerung der Tagesmüdigkeit. Über die Psychoedukation und die Bearbeitung dysfunktionaler Gedanken sowie über eine Wiederaufnahme ihrer vernachlässigten sozialen Aktivitäten gelang es ihr, sich weniger auf ihren Schlaf zu fokussieren und die Lebensqualität deutlich zu verbessern. Sie erlernte auch Achtsamkeit, hatte jedoch nicht den Eindruck, dass dadurch ihr Schlaf positiv beeinflusst wird. Allerdings stellte sie fest, dass sie mit gelegentlich noch auftretenden schlechten Nächten gelassener umgehen konnte.

\section{Komorbiditäten beachten}

Abschließend sei darauf hingewiesen, dass bei Schlafstörungen, die mit anderen psychischen Störungen assoziiert sind, die Behandlung dieser komorbiden Störungen mit beachtet und gegebenenfalls parallel mitbehandelt werden muss. Eine Antriebsförderung bei depressiven Patienten, die Verbesserung sozialer Kompetenzen bei sozialphoben Patienten oder eine Bearbeitung von Störungen der Emotionsregulation kann notwendig sein, um die hier dargestellten Maßnahmen zur Schlafverbesserung überhaupt erst umsetzbar zu machen. 


\section{KERNAUSSAGEN}

- Schlafstörungen ohne organische Ursache lassen sich unterteilen in Einschlaf- und/oder Durchschlafstörungen mit oft zu frühem Erwachen, die den Betroffenen unerholt und übermüdet in den Tag starten lassen.

- Sie müssen abgegrenzt werden von Schlafbeeinträchtigungen durch organische Ursachen wie etwa Herzinsuffizienzen, chronisch-obstruktiven Lungenerkrankungen, ein obstruktives oder zentrales Schlafapnoe-Syndrom, ein Syndrom der Unruhigen Beine (Restless-LegsSyndrom), eine Narkolepsie oder von den Parasomnien.

- Mit der Chronifizierung der Beschwerden gewinnen die aufrechterhaltenden Bedingungen der Insomnien gegenüber den Entstehungsbedingungen die Überhand.

- Die Kognitive Verhaltenstherapie der Insomnie (CBT-I) bietet ein gutes Störungsmodell und erfolgreich erprobte Interventionen zur Verbesserung der Störung.

- Kernelemente der Therapie sind Psychoedukation, schlafhygienische Maßnahmen, der Einsatz von Entspannungsverfahren, Stimuluskontrolle, Schlafrestriktion und die Bearbeitung dysfunktionaler Kognitionen zum Schlaf.

- Trotz des erfolgreichen Einsatzes der CBT-I wird sie gegenüber medikamentösen Maßnahmen viel zu selten eingesetzt. Gerade die Nebenwirkungen, das Suchtpotenzial mancher Schlafmittel und der häufige Wirkverlust der Medikationen beim langfristigen Gebrauch sollten Anlass sein, die Anwendung der CBT-I zu steigern.

Interessenkonflikt

\section{Erklärung zu finanziellen Interessen}

Forschungsförderung erhalten: ja, von einer anderen Institution (Pharma- oder Medizintechnikfirma usw.); Honorar/geldwerten Vorteil für Referententätigkeit erhalten: ja, von einer anderen Institution (Pharma- oder Medizintechnikfirma usw.); Bezahlter Berater/interner Schulungsreferent/ Gehaltsempfänger: nein; Patent/Geschäftsanteile/Aktien (Autor/Partner, Ehepartner, Kinder) an Firma (Nicht-Sponsor der Veranstaltung): nein; Patent/Geschäftsanteile/Aktien (Autor/Partner, Ehepartner, Kinder) an Firma (Sponsor der Veranstaltung): nein.

Erklärung zu nichtfinanziellen Interessen

Die Autorinnen/Autoren geben an, dass kein Interessenkonflikt besteht. Klaus Junghanns ist Mitglied der DGSM und der DGPPN.

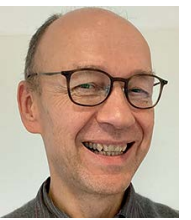

\section{Klaus Junghanns}

Prof. Dr. Jahrgang 1957. Studium der Humanmedizin und Philosophie in Hamburg, Facharzt für Neurologie und Psychiatrie, Zusatzbezeichnung Psychotherapie. Promotion 1985. Oberarzt in der Klinik für Psychiatrie und Psychotherapie der Universität zu Lübeck seit 1997. Habilitation im Bereich

Psychiatrie 2006, apl. Professur für Psychiatrie und Psychotherapie seit 2011. Forschungsschwerpunkte: Suchtmedizin und Schlafforschung.

\section{Korrespondenzadresse}

Prof. Dr. MA Klaus Junghanns

Klinik für Psychiatrie und Psychotherapie

Universität zu Lübeck

Ratzeburger Allee 160

23538 Lübeck

Deutschland

Klaus.Junghanns@uksh.de

\section{Wissenschaftlich verantwortlich gemäß}

\section{Zertifizierungsbestimmungen}

Wissenschaftlich verantwortlich gemäß Zertifizierungsbestimmungen für diesen Beitrag ist Prof. Dr. med. Klaus Junghanns, Lübeck.

\section{Zitierweise für diesen Artikel}

Psychother Psych Med 2020; 70: 519-532

Dieser Beitrag ist eine aktualisierte Version des Artikels: Junghanns K. Psychotherapie der Schlafstörungen. PSYCH up2date 2020; 14 (5): 419-432

\section{Literatur}

[1] Thünker J, Pietrowsky R. Alpträume. Göttingen: Hogrefe; 2011

[2] Born J, Wilhelm I. System consolidation of memory during sleep. Psychol Res 2012; 76: 192-203

[3] Besedovsky L, Lange T, Haack M. The Sleep-Immune Crosstalk in Health and Disease. Physiol Rev 2019; 99: $1325-1380$

[4] Backhaus J, Junghanns K, Born J et al. Impaired declarative memory consolidation during sleep in patients with primary insomnia: Influence of sleep architecture and nocturnal cortisol release. Biol Psych 2006; 60: 1324-1330

[5] Reinberg AE. Chronobiologie médicale, Chronothérapeutique. Paris: Flammarion; 2003

[6] Uber C, Ancoli-Israel S, Chesson A et al. The AASM Manual for the Scoring of Sleep and Associated Events. Darien IL. USA: American Academy of Sleep Medicine. 2012 
[7] Späth-Schwalbe E, Hundenborn C, Kern W et al. Nocturnal wakefulness inhibits growth hormone $(\mathrm{GH})$-releasing hormone-induced GH secretion. J Clin Endocrinol Metabol 1995; 80: 214-219

[8] Born J, Wagner U. Memory consolidation during sleep: role of cortisol feedback. Ann N Y Acad Sci 2004; 1032: 198-201

[9] Späth-Schwalbe E, Uthgenannt D, Voget G et al. Corticotropin-releasing hormone-induced adrenocorticotropin and cortisol secretion depends on sleep and wakefulness. J Clin Endocrinol Metabol 1993; 77: 1170-1173

[10] Fries E, Dettenborn L, Kirschbaum C. The cortisol awakening response (CAR): facts and future directions. Int J Psychophysiol 2009; 72: 67-73

[11] American Psychiatric Association, APA. Diagnostic Criteria from DSM-5. Arlington, VA: APA; 2013

[12] Morin CM. Insomnia. New York, USA: Guilford Press; 1993

[13] Riemann D. Backhaus. J. Behandlung von Schlafstörungen. Weinheim: Beltz PVU. 1996

[14] Edinger JD, Carney CE. Overcoming Insomnia. Oxford: University Press; 2008

[15] Crönlein T. Primäre Insomnie. Göttingen: Hogreve. 2013

[16] Spiegelhalder K, Hajak G, Riemann D. Nichtorganisch bedingte Schlafstörungen (ICD-10 F5. In: Voderholzer U, Hohagen F, Hrsg. Therapie psychischer Erkrankungen, State of the Art. 13. Aufl. München: Urban \& Fischer; 2018: 357-364
[17] van Straten A et al. Cognitive and behavioral therapies in the treatment of insomnia. A meta-analysis. Sleep Med Rev 2018; 38: 3-16

[18] Riemann D, Baum E, Cohrs S et al. S3-Leitlinie Nicht erholsamer Schlaf/Schlafstörungen. Kapitel „Insomnie bei Erwachsenen “. Somnologie 2017; 21: 2-44

[19] Spielman A, Caruso L, Glovinsky P. A behavioral perspective on insomnia treatment. Psychiatr Clin North Am 1987; 10: 541-553

[20] Wampold BE, Imel ZE. The great psychotherapy debate. $2^{\text {nd }}$. Edition New York: Taylor and Francis; 2015

[21] Gumenyuk V, Belcher R, Roth T. Differential sleep, sleepiness, and neurophysiology in the insomnia phenotypes of shift work disorder. Sleep 2015; 38: 119-126

Bibliografie

Psychother Psych Med 2020; 70: 519-532

DOI $10.1055 / \mathrm{a}-1254-4399$

ISSN $\quad 0937-2032$

(c) 2020. Thieme. All rights reserved.

Georg Thieme Verlag KG, Rüdigerstraße 14,

70469 Stuttgart, Germany 


\section{Punkte sammeln auf CME.thieme.de}

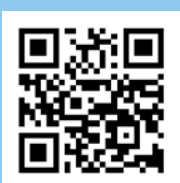

Diese Fortbildungseinheit ist bis zu 12 Monate online für die Teilnahme verfügbar.

Den genauen Einsendeschluss finden Sie beim Modul auf https://eref.thieme.de/CXFN7L1.

Sollten Sie Fragen zur Online-Teilnahme haben, finden Sie unter https://cme.thieme.de/hilfe

eine ausführliche Anleitung. Wir wünschen viel Erfolg beim Beantworten der Fragen!

Unter https://eref.thieme.de/CXFN7L1 oder über den QR-Code kommen Sie direkt zum Artikel.

\section{Frage 1}

Was trifft zu?

A Die Diagnose der nichtorganischen Insomnie fußt auf schlafpolysomnografischen Kriterien.

B Neben Schlafstörungen wird für die Diagnosestellung eine Beeinträchtigung am Tag gefordert.

C Die Schlafdauer ist ein wesentliches Kriterium für die Diagnose der Insomnie.

D Tiefschlafmangel ist ein diagnostisches Kriterium für Insomnie.

E Tiefschlaf ist typisch in den frühen Morgenstunden.

\section{Frage 2}

Was trifft nicht zu?

A Dynamische Träume haben wir v. a. im REM-Schlaf.

B Gesunde Schläfer durchlaufen 4-6 ultradiane Zyklen in einer Nacht.

C Schwimmende Augenbewegungen sind charakteristisch für Tiefschlaf.

D Im REM-Schlaf ist die Motorik zentralnervös gehemmt.

E Sakkadische Augenbewegungen im Schlaf sind typisch für REM.

\section{Frage 3}

Was trifft zu?

A In der zweiten Nachthälfte ist die Cortisol-Sekretion besonders niedrig.

B Die Melatonin-Sekretion ist in der zweiten Nachthälfte besonders hoch.

C Die Körperkerntemperatur fällt zum Morgen hin ab.

D REM-Schlaf tritt besonders stark in der zweiten Nachthälfte auf.

E Tiefschlaf fördert das Vergessen.

\section{Frage 4}

Was trifft nicht zu?

A Schlafstörungen treten oft bei Depressionen auf.

B Mirtazapin fördert häufig eine Restless-Legs-Symptomatik.

C Die meisten Antidepressiva sind REM-suppressiv.

D Internistische Erkrankungen gehen oft mit Insomnien einher.

E Alkohol fördert das Ein- und Durchschlafen.

\section{Frage 5}

Was trifft zu?

A Wer mehr als dreimal in der Nacht wach wird, hat eine Insomnie.

B Wer täglich neun bis zehn Stunden schläft, hat eine Hypersomnie.

C Das Auftreten von Schlafstörungen an mindestens drei Nächten pro Woche ist ein diagnostisches Kriterium der Insomnie.

D Bei einem Schlafapnoe-Syndrom werden typischerweise Insomnien beklagt.

E Narkolepsie ist nicht mit insomnischen Beschwerden verbunden.

\section{Frage 6}

Was trifft nicht zu?

A In der CBT-I geht man von einem Teufelskreis aus, wobei sich u. a. emotionale und psychovegetative Aspekte gegenseitig beeinflussen.

B Zu den schlafhygienischen Maßnahmen gehört der Verzicht auf Alkohol und regelmäßige Bettzeiten.

C Anstelle von Entspannungsverfahren sollte abendlicher Sport zur Schlafförderung eingesetzt werden.

D Eine stabile Aufstehzeit ist für Schlafverbesserung wichtiger als die Zeit für das Zubettgehen.

E Schlafhygienische Maßnahmen sollten individuell erprobt und abgestimmt werden.

\section{Frage 7}

Was trifft zu?

A Durch Stimuluskontrolle soll besonders die Einschlafstörung verbessert werden.

B Entspannungsverfahren sollten im Bett nicht angewandt werden.

C Bei ausgeprägten Schlafstörungen sollte eine Bettzeit von 7 Stunden strikt eingehalten werden.

D Angenehme abendliche soziale Aktivitäten sollten für die Zeit der CBT-I ausgesetzt werden.

E Fernsehen von Spielfilmen sollte bei Einschlafstörungen eingesetzt werden. 


\section{Punkte sammeln auf CME.thieme.de}

\section{Fortsetzung ...}

\section{Frage 8}

Was trifft nicht zu?

A Stimuluskontrolle geht davon aus, dass eine klassische Konditionierung die Einschlafprobleme wesentlich mitbeeinflusst.

B Eine schlafpolysomnografische Untersuchung bei insomnischen Beschwerden ist kontraindiziert.

C Progressive Muskelentspannung mit Phantasiereise zielt auch auf eine kognitive Entspannung ab.

D Die Phantasiereise bezieht sich auf die möglichst plastische Imagination eines Ortes der Entspannung.

E Schlafrestriktion zielt auf eine Verbesserung der Schlafeffizienz.

\section{Frage 9}

Was trifft zu?

A Schlafentzug bei Depressionsbehandlung heißt bei Insomniebehandlung Schlafrestriktion.

B Schlafrestriktion zielt darauf ab, den Schlaf nach Mitternacht zu kupieren.

C Eine Ausweitung der Bettzeit unter Schlafrestriktion wird von der Schlafeffizienz abhängig gemacht.

D Schlafrestriktion ist besonders bei Einschlafstörungen sinnvoll.

E Schlafrestriktion führt zu einer Stimmungsaufhellung.

\section{Frage 10}

Was trifft nicht zu?

A Katastrophisierung der Schlafstörung ist typisch für dysfunktionales Denken.

B Dysfunktionale Gedanken wirken aufrechterhaltend auf die Insomnie.

C Für die Disputation dysfunktionaler Gedanken sollten die Schlafprotokolle genutzt werden.

D Schlafpolysomnographische Untersuchungen helfen oft, dysfunktionale Gedanken zu relativieren.

E Der Einsatz eines Sorgenstuhls verfestigt dysfunktionales Denken. 